Georgian Mathematical Journal

1(1994), No. 3, 287-302

\title{
ON SOME PROPERTIES OF MULTIPLE CONJUGATE TRIGONOMETRIC SERIES
}

\author{
D. LELADZE
}

\begin{abstract}
We have obtained the estimate in the terms of partial and mixed moduli of continuity of deviation of Cesáro $(C, \quad)$ means $(=$ $\left.\left(\alpha_{1}, \ldots, \alpha_{n}\right), \alpha_{i} \in \mathbb{R}, \alpha_{i}>-1, i=\overline{1, n}\right)$ of the sequence of rectangular partial sums of $n$-multiple $(n>1)$ conjugate trigonometric series from $n$-multiple truncated conjugate function. This estimate implies the result on the $m_{\lambda}$-convergence $(\lambda \geq 1)$ of $(C, \quad)$ means $\left(\alpha_{i}>0, i=\right.$ $\overline{1, n})$, provided that the essential conditions are imposed on the partial moduli of continuity. Finally, it is shown, that the $m_{\lambda}$-convergence cannot be replaced by ordinary convergence.
\end{abstract}

1. Let $f \in L\left([-\pi ; \pi]^{n}\right), n \in \mathbb{N}, n>1$, be a function, $2 \pi$-periodic in each variable, $\sigma_{n}[f]$ its $n$-multiple trigonometric Fourier series, and $\bar{\sigma}_{n}[f]$ its conjugate series with respect to $n$ variables (see, e.g., [1]).

We set

$$
\begin{gathered}
\mathbf{m}=\left(m_{1}, \ldots, m_{n}\right) \quad\left(m_{i} \in \mathbb{N}, i=\overline{1, n}\right) \\
\mathbf{x}=\left(x_{1}, \ldots, x_{n}\right) \quad\left(x_{i} \in \mathbb{R}, i=\overline{1, n}\right) ; \\
\boldsymbol{\alpha}=\left(\alpha_{1}, \ldots, \alpha_{n}\right) \quad\left(\alpha_{i} \in \mathbb{R}, \alpha_{i}>-1, i=\overline{1, n}\right) ; \quad \mathbf{M}=\{1,2, \ldots, n\} .
\end{gathered}
$$

By $M_{j}$ we denote the set of all subsets of $M$ with $j$ elements, by $M^{(A)}$ a set $M \backslash A(A \subset M)$, and by $M_{j}^{(A)}$ the set of all subsets of $M^{(A)}$ with $j$ elements.

For $B=\left\{i_{i}, \ldots, i_{k}\right\} \subset M$ we define $m(B)=\left\{1 / m_{i_{1}}, \ldots, 1 / m_{i_{k}}\right\}$ and the

1991 Mathematics Subject Classification. 42B08. 
truncated conjugate function with respect to the corresponding variables

$$
\begin{gathered}
\bar{f}_{m(B)}(\mathbf{x})= \\
=\frac{1}{(-2 \pi)^{k}} \int_{1 / m_{i_{1}}}^{\pi} \ldots \int_{1 / m_{i_{k}}}^{\pi}\left(\Delta_{k, s_{i_{k}}}\left(\Delta_{k-1, s_{i_{k-1}}}\left(\ldots\left(\Delta_{1, s_{i_{1}}}(f ; \mathbf{x})\right) \ldots\right)\right)\right) \times \\
\times \prod_{j=1}^{k} \operatorname{ctg} \frac{s_{i_{j}}}{2} d s_{i_{1}} \ldots d s_{i_{k}}, \quad \bar{f}_{\mathbf{m}}(\mathbf{x})=\bar{f}_{m(M)}(\mathbf{x}),
\end{gathered}
$$

where

$$
\begin{aligned}
\Delta_{i, h}(f, \mathbf{x}) & =f\left(x_{1}, \ldots, x_{i-1}, x_{i}+h, x_{i+1}, \ldots, x_{n}\right)- \\
& -f\left(x_{1}, \ldots, x_{i-1}, x_{i}-h, x_{i+1}, \ldots, x_{n}\right), \quad i=\overline{1, n} .
\end{aligned}
$$

For $f \in L^{q}\left([-\pi ; \pi]^{n}\right), 1 \leq q \leq+\infty\left(L^{\infty}=C\right)$, we consider its mixed modulus of continuity

$$
\begin{gathered}
\omega_{B}(m(B) ; f)_{L^{q}}= \\
=\sup _{\left|h_{1}\right|<1 / m_{i_{1}}, \ldots,\left|h_{k}\right|<1 / m_{i_{k}}}\left\|\Delta_{k}^{h_{k}}\left(\Delta_{k-1}^{h_{k-1}}\left(\ldots\left(\Delta_{1}^{h_{1}}(f ; \mathbf{x})\right) \ldots\right)\right)\right\|_{L^{q}}
\end{gathered}
$$

where $\Delta_{i}^{h}(f ; \mathbf{x})=f\left(x_{1}, \ldots, x_{i-1}, x_{i}+h, x_{i+1}, \ldots, x_{n}\right)-f\left(x_{1}, \ldots, x_{n}\right)$.

Let $\bar{\sigma}_{\mathbf{m}}^{\boldsymbol{\alpha}}(\mathbf{x} ; f)$ be Cesáro means of $\bar{\sigma}_{n}[f]$.

In the sequel by $A, B, A_{1}, B_{1}, C(\alpha), C(\beta), C(\alpha, \beta)$, etc. we will denote, in general, different positive constants.

Finally, we set

$$
\lambda(k, \beta)= \begin{cases}k^{-\beta}, & -1<\beta<0 \\ \ln (k+1), & \beta=0, \\ 1, & \beta>0, \quad(k=1,2, \ldots) .\end{cases}
$$

In the present paper we give the estimate of the deviation of $n$-multiple Cesáro means of the sequence of rectangular partial sums of $\bar{\sigma}_{n}[f]$ from $\bar{f}_{\mathbf{m}}$ in the norm of $L^{q}, q \in[1 ;+\infty]\left(L^{\infty}=C\right)$, in terms of partial and mixed moduli of continuity of $f$. This result generalizes the corresponding result of L.Zhizhiashvili (see [1]).

From this estimate ensues the result on the Cesáro summability of the sequence of rectangular partial sums of $\bar{\sigma}_{n}[f]$ and then the correctness of this result is shown.

2. The following is true: 
Theorem 1. If $f \in L^{q}\left([-\pi ; \pi]^{n}\right), q \in[1 ;+\infty]\left(L^{\infty}=C\right)$, then

$$
\begin{gathered}
\left\|\bar{\sigma}_{\mathbf{m}}^{\boldsymbol{\alpha}}(\mathbf{x} ; f)-\bar{f}_{\mathbf{m}}(\mathbf{x})\right\|_{L^{q}} \leq C(\boldsymbol{\alpha})\left\{\sum_{k=1}^{n-1} \sum_{B \in M_{k}} \omega_{B}(m(B) ; f)_{L^{q}} \times\right. \\
\times \quad \prod_{\substack{j=1 \\
\left\{i_{1}, \ldots, i_{k}\right\} \in M_{k}}}^{k} \lambda\left(m_{i_{j}}, \alpha_{i_{j}}\right)+\sum_{i=1}^{n-1} \sum_{k=1}^{n-i} \sum_{A \in M_{i}} \sum_{B \in M_{k}^{(A)}} \omega_{B}\left(m(B) ; \bar{f}_{m(A)}\right)_{L^{q}} \times \\
\left.\times \prod_{\substack{j=1 \\
\left\{t_{1}, \ldots, t_{k}\right\} \in M_{k}^{(A)}}} \lambda\left(m_{t_{j}}, \alpha_{t_{j}}\right)+\prod_{i=1}^{n} \lambda\left(m_{i}, \alpha_{i}\right) \omega_{M}(m(M) ; f)_{L^{q}}\right\} .
\end{gathered}
$$

Proof. For simplicity, we will prove the theorem in the case $n=2$ which is typical. We will use the method of L.Zhizhiashvili ([1], p. 160-191), this method proving to be true for $n \geq 3$.

Let $\omega\left(\delta_{1}, \delta_{2} ; f\right)_{L^{p}}$ be the mixed modulus of continuity of the function $f(x, y), x, y \in \mathbb{R}$, with respect to two variables. By $\omega_{i}(\delta ; f)_{L^{q}}(i \in \mathbb{N}, \delta \in$ $\mathbb{R}^{+}$), as usual, we denote the modulus of continuity of $f$ in $L^{q}$ with respect to the corresponding variable. Let $m, n \in \mathbb{N}, \alpha, \beta \in \mathbb{R}, \alpha, \beta>-1, \bar{f}_{m, n}(x, y)$ be the two-dimensional truncated conjugate function and $\bar{\sigma}_{m n}^{\alpha, \beta}(x, y ; f)$ be the Cesáro means of the double conjugate series $\bar{\sigma}_{2}[f]$.

We have ([1], p.187-188):

$$
\begin{aligned}
\bar{\sigma}_{m n}^{\alpha, \beta}(x, y ; f) & =1 / \pi^{2} \int_{0}^{\pi / m} \int_{0}^{\pi / n} \psi_{x, y}(s, t ; f) \bar{K}_{m}^{\alpha}(s) \bar{K}_{n}^{\beta}(t) d t d s+ \\
& +1 /\left(2 \pi^{2}\right) \int_{0}^{\pi / m} \int_{\pi / n}^{\pi} \psi_{x, y}(s, t ; f) \operatorname{ctg} \frac{t}{2} \bar{K}_{m}^{\alpha}(s) d t d s+ \\
& +1 / \pi^{2} \int_{0}^{\pi / m} \int_{\pi / n}^{\pi} \psi_{x, y}(s, t ; f) \bar{K}_{m}^{\alpha}(s) H_{n, 1}^{\beta}(t) d t d s+ \\
& +1 / \pi^{2} \int_{0}^{\pi / m} \int_{\pi / n}^{\pi} \psi_{x, y}(s, t ; f) \bar{K}_{m}^{\alpha}(s) H_{n, 2}^{\beta}(t) d t d s+ \\
& +1 /\left(2 \pi^{2}\right) \int_{\pi / m}^{\pi} \int_{0}^{\pi / n} \psi_{x, y}(s, t ; f) \operatorname{ctg} \frac{s}{2} \bar{K}_{n}^{\beta}(t) d t d s+ \\
& +1 / \pi^{2} \int_{\pi / m}^{\pi} \int_{0}^{\pi / n} \psi_{x, y}(s, t ; f) H_{m, 1}^{\alpha}(s) \bar{K}_{n}^{\beta}(t) d t d s+ \\
& +1 / \pi^{2} \int_{\pi / m}^{\pi} \int_{0}^{\pi / n} \psi_{x, y}(s, t ; f) H_{m, 2}^{\alpha}(s) \bar{K}_{n}^{\beta}(t) d t d s+ \\
& +1 /\left(4 \pi^{2}\right) \int_{\pi / m}^{\pi} \int_{\pi / n}^{\pi} \psi_{x, y}(s, t ; f) \operatorname{ctg} \frac{s}{2} \operatorname{ctg} \frac{t}{2} d t d s+
\end{aligned}
$$




$$
\begin{aligned}
& +1 /\left(2 \pi^{2}\right) \int_{\pi / m}^{\pi} \int_{\pi / n}^{\pi} \psi_{x, y}(s, t ; f) \operatorname{ctg} \frac{s}{2} H_{n, 1}^{\beta}(t) d t d s+ \\
& +1 /\left(2 \pi^{2}\right) \int_{\pi / m}^{\pi} \int_{\pi / n}^{\pi} \psi_{x, y}(s, t ; f) \operatorname{ctg} \frac{s}{2} H_{n, 2}^{\beta}(t) d t d s+ \\
& +1 /\left(2 \pi^{2}\right) \int_{\pi / m}^{\pi} \int_{\pi / n}^{\pi} \psi_{x, y}(s, t ; f) \operatorname{ctg} \frac{t}{2} H_{m, 1}^{\alpha}(s) d t d s+ \\
& +1 / \pi^{2} \int_{\pi / m}^{\pi} \int_{\pi / n}^{\pi} \psi_{x, y}(s, t ; f) H_{m, 1}^{\alpha}(s) H_{n, 1}^{\beta}(t) d t d s+ \\
& +1 / \pi^{2} \int_{\pi / m}^{\pi} \int_{\pi / n}^{\pi} \psi_{x, y}(s, t ; f) H_{m, 1}^{\alpha}(s) H_{n, 2}^{\beta}(t) d t d s+ \\
& +1 /\left(2 \pi^{2}\right) \int_{\pi / m}^{\pi} \int_{\pi / n}^{\pi} \psi_{x, y}(s, t ; f) \operatorname{ctg} \frac{t}{2} H_{m, 2}^{\alpha}(s) d t d s+ \\
& +1 / \pi^{2} \int_{\pi / m}^{\pi} \int_{\pi / n}^{\pi} \psi_{x, y}(s, t ; f) H_{m, 2}^{\alpha}(s) H_{n, 1}^{\beta}(t) d t d s+ \\
& +1 / \pi^{2} \int_{\pi / m}^{\pi} \int_{\pi / n}^{\pi} \psi_{x, y}(s, t ; f) H_{m, 2}^{\alpha}(s) H_{n, 2}^{\beta}(t) d t d s= \\
& =\sum_{j=1}^{16} P_{m n}^{(j)}(x, y ; f),
\end{aligned}
$$

where

$$
\begin{aligned}
\psi_{x, y}(s, t ; f) & =f(x+s, y+t)-f(x-s, y+t)- \\
& -f(x+s, y-t)+f(x-s, y-t)
\end{aligned}
$$

and $H_{m, 1}^{\alpha}(s), H_{m, 2}^{\alpha}(s)$ are the summands of the conjugate Fejér kernel $\bar{K}_{m}^{\alpha}(s)$ (see [2], pp.157-160)

$$
\begin{aligned}
& \bar{K}_{m}^{\alpha}(s)=\frac{1}{2} \operatorname{ctg} \frac{s}{2}+H_{m, 1}^{\alpha}(s)+H_{m, 2}^{\alpha}(s), \\
& H_{m, 1}^{\alpha}(s)=-\frac{\cos \left(\left(m+\frac{1}{2}+\frac{\alpha}{2}\right) s-\frac{\pi \alpha}{2}\right)}{A_{m}^{\alpha}\left(2 \sin \frac{s}{2}\right)^{1+\alpha}} .
\end{aligned}
$$

Besides,

$$
\begin{gathered}
\left|\bar{K}_{m}^{\alpha}(s)\right| \leq C(\alpha) m, \quad|s| \leq \pi \\
\left|H_{m, 2}^{\alpha}(s)\right| \leq \frac{C(\alpha)}{m^{2} s^{3}}, \quad \pi / m \leq|s| \leq \pi, \quad m>1 .
\end{gathered}
$$

The estimate (7) is more precise than the estimate (2.1.14) in [1] and it can be proved by arguments analogous to those in [2], pp.157-160. 
From (2) we obtain

$$
\begin{gathered}
\bar{\sigma}_{m n}^{\alpha, \beta}(x, y ; f)-\bar{f}_{m n}(x, y)=\bar{\sigma}_{m n}^{\alpha, \beta}(x, y ; f)-P_{m n}^{(8)}(x, y ; f)= \\
=\sum_{k=1}^{16} P_{m n}^{(k)}(x, y ; f),
\end{gathered}
$$

where ' indicates that the eighth member is omitted (the replacement of $1 / m(1 / n)$ in $\bar{f}_{m n}(x, y)$ by $\pi / m(\pi / n)$ does not matter $)$.

In the sequel we will use the inequality (see [3], p.179)

$$
\left\{\int_{a}^{b}\left|\int_{c}^{d} f(x, y) d y\right|^{q} d x\right\}^{1 / q} \leq \int_{c}^{d}\left\{\int_{a}^{b}|f(x, y)|^{q} d x\right\}^{1 / q} d y
$$

Taking into account (6) and (9), we obtain

$$
\left\|P_{m n}^{(1)}(x, y ; f)\right\|_{L^{q}} \leq C(\alpha, \beta)\left\{\omega_{1}(1 / m ; f)_{L^{q}}+\omega_{2}(1 / n ; f)_{L^{q}}\right\} .
$$

It is easy to see, that

$$
\begin{gathered}
P_{m n}^{(2)}(x, y ; f)=-\frac{1}{\pi} \int_{0}^{\pi / m}\left[\bar{f}_{n}^{(2)}(x+s, y)-\bar{f}_{n}^{(2)}(x-s, y)\right] \bar{K}_{m}^{\alpha}(s) d s \\
P_{m n}^{(5)}(x, y ; f)=-\frac{1}{\pi} \int_{0}^{\pi / n}\left[\bar{f}_{m}^{(1)}(x, y+t)-\bar{f}_{m}^{(1)}(x, y-t)\right] \bar{K}_{n}^{\beta}(t) d t
\end{gathered}
$$

where

$$
\begin{gathered}
\bar{f}_{m}^{(1)}(x, y)=-\frac{1}{2 \pi} \int_{\pi / m}^{\pi}[f(x+s, y)-f(x-s, y)] \operatorname{ctg} \frac{s}{2} d s \\
\bar{f}_{n}^{(2)}(x, y)=-\frac{1}{2 \pi} \int_{\pi / n}^{\pi}[f(x, y+t)-f(x, y-t)] \operatorname{ctg} \frac{t}{2} d t .
\end{gathered}
$$

Hence, using again (6) and (9), we obtain:

$$
\begin{gathered}
\left\|P_{m n}^{(2)}(x, y ; f)\right\|_{L^{q}} \leq C(\alpha, \beta) \omega_{1}\left(1 / m ; \bar{f}_{n}^{(2)}\right)_{L^{q}} ; \\
\left\|P_{m n}^{(5)}(x, y ; f)\right\|_{L^{q}} \leq C(\alpha, \beta) \omega_{2}\left(1 / n ; \bar{f}_{m}^{(1)}\right)_{L^{q}} .
\end{gathered}
$$

Now, it is easy to see that for estimation of $P_{m n}^{(3)}(x, y ; f)$ it suffices to estimate the integral

$$
I(m, n)=n^{-\beta} \int_{0}^{\pi / m} \int_{\pi / n}^{\pi} \psi_{x, y}(s, t ; f) \bar{K}_{m}^{\alpha}(s) \omega_{\beta}(t) \cos n t d t d s,
$$

where

$$
\omega_{\beta}(t)=\frac{\cos \frac{1+\beta}{2} t}{\left(\sin \frac{t}{2}\right)^{1+\beta}}
$$


We have

$$
\begin{aligned}
& 2 I(m, n)=n^{-\beta}\left\{\int_{0}^{\pi / m} \int_{\pi / n}^{\pi}\left[\psi_{x, y}(s, t ; f)-\psi_{x, y}(s, t+\pi / n ; f)\right] \times\right. \\
& \times \bar{K}_{m}^{\alpha}(s) \omega_{\beta}(t) \cos n t d t d s+\int_{0}^{\pi / m} \int_{\pi / n}^{\pi} \psi_{x, y}(s, t+\pi / n ; f)\left[\omega_{\beta}(t)-\right. \\
& \left.\quad-\omega_{\beta}(t+\pi / n)\right] \bar{K}_{m}^{\alpha}(s) \cos n t d t d s+ \\
& +\int_{0}^{\pi / m} \int_{\pi-\pi / n}^{\pi} \psi_{x, y}(s, t+\pi / n ; f) \bar{K}_{m}^{\alpha}(s) \omega_{\beta}(t+\pi / n) \cos n t d t d s- \\
& \left.-\int_{0}^{\pi / m} \int_{0}^{\pi / n} \psi_{x, y}(s, t+\pi / n ; f) \bar{K}_{m}^{\alpha}(s) \omega_{\beta}(t+\pi / n) \cos n t d t d s\right\} .
\end{aligned}
$$

Now we note that (see [1], p.56, (2.1.18))

$$
\left|\omega_{\beta}(t)-\omega_{\beta}(t+\pi / n)\right| \leq C(\beta) /\left(n t^{2+\beta}\right), \quad \frac{\pi}{n} \leq t \leq \pi .
$$

(6) and (15) yield $\|I(m, n)\|_{L^{q}} \leq C(\alpha, \beta) \lambda(n, \beta) \omega_{2}(1 / n ; f)_{L^{q}}$ and hence

$$
\left\|P_{m n}^{(3)}(x, y ; f)\right\|_{L^{q}} \leq C(\alpha, \beta) \lambda(n, \beta) \omega_{2}(1 / n ; f)_{L^{q}} .
$$

Analogously

$$
\left\|P_{m n}^{(6)}(x, y ; f)\right\|_{L^{q}} \leq C(\alpha, \beta) \lambda(m, \alpha) \omega_{1}(1 / m ; f)_{L^{q}} .
$$

Furthermore, using (6),(7) and (9), we obtain

$$
\begin{gathered}
\left\|P_{m n}^{(k)}(x, y ; f)\right\|_{L^{q}} \leq C(\alpha, \beta)\left\{\omega_{1}(1 / m ; f)_{L^{q}}+\omega_{2}(1 / n ; f)_{L^{q}}\right\} \\
(k=4,7,16) .
\end{gathered}
$$

Analogously, taking into account that

$$
\begin{aligned}
& P_{m n}^{(9)}(x, y ; f)=-\frac{1}{\pi} \int_{\pi / n}^{\pi}\left[\bar{f}_{m}^{(1)}(x, y+t)-\bar{f}_{m}^{(1)}(x, y-t)\right] H_{n, 1}^{\beta}(t) d t \\
& P_{m n}^{(11)}(x, y ; f)=-\frac{1}{\pi} \int_{\pi / m}^{\pi}\left[\bar{f}_{n}^{(2)}(x+s, y)-\bar{f}_{n}^{(2)}(x-s, y)\right] H_{m, 1}^{\alpha}(s) d s
\end{aligned}
$$

we can prove

$$
\begin{gathered}
\left\|P_{m n}^{(9)}(x, y ; f)\right\|_{L^{q}} \leq C(\alpha, \beta) \lambda(n, \beta) \omega_{2}\left(1 / n ; \bar{f}_{m}^{(1)}\right)_{L^{q}} \\
\left\|P_{m n}^{(11)}(x, y ; f)\right\|_{L^{q}} \leq C(\alpha, \beta) \lambda(m, \alpha) \omega_{1}\left(1 / m ; \bar{f}_{n}^{(2)}\right)_{L^{q}}
\end{gathered}
$$

Using the same arguments and applying (6), (7) and (9), we can prove

$$
\left\|P_{m n}^{(10)}(x, y ; f)\right\|_{L^{q}} \leq C(\alpha, \beta) \omega_{2}\left(1 / n ; \bar{f}_{m}^{(1)}\right)_{L^{q}}
$$




$$
\left\|P_{m n}^{(14)}(x, y ; f)\right\|_{L^{q}} \leq C(\alpha, \beta) \omega_{1}\left(1 / m ; \bar{f}_{n}^{(2)}\right)_{L^{q}} .
$$

Now we observe that the following lemma holds true (see [1], p.160, Lemma 10):

Lemma 1. Let $f \in L^{q}\left([-\pi ; \pi]^{2}\right), 1 \leq q \leq+\infty$, and

$$
\begin{aligned}
\phi_{x, y}(s, t ; f) & =f(x+s, y+t)+f(x-s, y+t)+f(x+s, y-t)+ \\
& +f(x-s, y-t)-4 f(x, y) .
\end{aligned}
$$

Then

$$
\begin{gathered}
\left\|m^{-\alpha} n^{-\beta} \int_{\pi / m}^{\pi} \int_{\pi / n}^{\pi} \phi_{x, y}(s, t ; f) \omega_{\alpha}(s) \omega_{\beta}(t) \cos _{\sin }^{\sin } \operatorname{sos}_{\cos }^{\sin } n t d t d s\right\|_{L^{q}}= \\
=O\left\{\lambda(m, \alpha) \lambda(n, \beta) \omega(1 / m, 1 / n ; f)_{L^{q}}+\lambda(m, \alpha) \omega_{1}(1 / m ; f)_{L^{q}}+\right. \\
\left.+\lambda(n, \beta) \omega_{2}(1 / n ; f)_{L^{q}}\right\} .
\end{gathered}
$$

There is another lemma in [1] (see p.171, Lemma 11), which can be corrected by means of (7) as follows:

Lemma 2. For $f \in L^{q}\left([-\pi ; \pi]^{2}\right), 1 \leq q \leq+\infty$, we have

$$
\begin{gathered}
\left\|m^{-\alpha} \int_{\pi / m}^{\pi} \int_{\pi / n}^{\pi} \phi_{x, y}(s, t ; f) \omega_{\alpha}(s) H_{n, 2}^{\beta}(t) \sin m s d t d s\right\|_{L^{q}}= \\
=O\left\{\lambda(m, \alpha) \omega_{1}(1 / m ; f)_{L^{q}}+\omega_{2}(1 / n ; f)_{L^{q}}\right\} ; \\
\| n^{-\beta} \int_{\pi / m}^{\pi} \int_{\pi / n}^{\pi} \phi_{x, y}(s, t ; f) \omega_{\beta}(t) H_{m, 2}^{\alpha}(s) \\
\quad \sin n t d t d s \|_{L^{q}}= \\
=O\left\{\lambda(n, \beta) \omega_{2}(1 / n ; f)_{L^{q}}+\omega_{1}(1 / m ; f)_{L^{q}}\right\} .
\end{gathered}
$$

The lemmas and (3), (5), (7) and (23) yield

$$
\begin{gathered}
\left\|P_{m n}^{(12)}(x, y ; f)+P_{m n}^{(13)}(x, y ; f)+P_{m n}^{(15)}(x, y ; f)\right\|_{L^{q}} \leq \\
\leq C(\alpha, \beta)\left\{\lambda(m, \alpha) \lambda(n, \beta) \omega(1 / m, 1 / n ; f)_{L^{q}}+\lambda(m, \alpha) \omega_{1}(1 / m ; f)_{L^{q}}+\right. \\
\left.+\lambda(n, \beta) \omega_{2}(1 / n ; f)_{L^{q}}\right\} .
\end{gathered}
$$

Finally, (2)-(24) yield

$$
\begin{gathered}
\left\|\bar{\sigma}_{m n}^{\alpha, \beta}(x, y ; f)-\bar{f}_{m n}(x, y)\right\|_{L^{q}} \leq \\
\leq C(\alpha, \beta)\left\{\lambda(m, \alpha) \lambda(n, \beta) \omega(1 / m, 1 / n ; f)_{L^{q}}+\lambda(m, \alpha) \omega_{1}(1 / m ; f)_{L^{q}}+\right. \\
+\lambda(n, \beta) \omega_{2}(1 / n ; f)_{L^{q}}+\lambda(m, \alpha) \omega_{1}\left(1 / m ; \bar{f}_{n}^{(2)}\right)_{L^{q}}+ \\
\left.+\lambda(n, \beta) \omega_{2}\left(1 / n ; \bar{f}_{m}^{(1)}\right)_{L^{q}}\right\}
\end{gathered}
$$

which is the formula (1) in the case $n=2$. 
Corollary. If $f \in C\left([-\pi ; \pi]^{n}\right)(n \geq 2)$ and

$$
\omega_{i}(\delta ; f)_{C}=o\left(1 / \ln ^{n-1}(1 / \delta)\right) \quad(i=\overline{1, n})
$$

as $\delta \rightarrow 0+$, then $\lim _{\mathbf{m}_{\lambda} \rightarrow \infty}\left\|\bar{\sigma}_{\mathbf{m}}^{\boldsymbol{\alpha}}(\mathbf{x} ; f)-\bar{f}_{\mathbf{m}}(\mathbf{x})\right\|_{C}=0, \boldsymbol{\alpha}=\left(\alpha_{1}, \ldots, \alpha_{n}\right), \alpha_{i}>0$ $(i=\overline{1, n}), \lambda \geq 1$.

Now we will prove that the $m_{\lambda}$-summability in the corollary is essential. Namely, the following result holds true:

Theorem 2. There exists a function $f \in C\left([-\pi ; \pi]^{n}\right)$ which satisfies (26) and

$$
\varlimsup_{\mathbf{m} \rightarrow \infty}\left|\bar{\sigma}_{\mathbf{m}}^{\boldsymbol{\alpha}}(\mathbf{0} ; f)-\bar{f}_{\mathbf{m}}(\mathbf{0})\right|=+\infty
$$

$\boldsymbol{\alpha}=\left(\alpha_{1}, \ldots, \alpha_{n}\right), \alpha_{i}>0(i=\overline{1, n}), \mathbf{0}=(0,0, \ldots, 0)$.

Proof. We will prove the theorem for $n=2$, this case being quite typical. First, let $\alpha, \beta \in(0 ; 1)$. We set

$$
\begin{aligned}
& m_{k}=2^{2^{2^{k}}}, \quad n_{k}=m_{k}^{m_{k}^{m_{k}^{m_{k}}}}, \quad(k \in \mathbb{N}) ; \\
& \ln ^{(2)} x=\ln \ln x, \quad \ln ^{(k)} x=\ln \ln ^{(k-1)} x, \quad k \in \mathbb{N}, \quad k \geq 3 ; \\
& g_{k}(x)= \begin{cases}\frac{1}{\ln \left(1 /\left(x-\pi /\left(6 m_{k}\right)\right)\right) \ln ^{(2)}\left(1 /\left(x-\pi /\left(6 m_{k}\right)\right)\right)}, & \frac{\pi}{6 m_{k}}<x \leq \frac{11 \pi}{60 m_{k}}, \\
\frac{1}{\ln \left(1 /\left(\pi /\left(5 m_{k}\right)-x\right)\right) \ln (2)\left(1 /\left(\pi /\left(5 m_{k}\right)-x\right)\right)}, & \frac{11 \pi}{60 m_{k}} \leq x<\frac{\pi}{5 m_{k}}, \\
0, & x \in(0 ; \pi) \backslash\left(\frac{\pi}{6 m_{k}} ; \frac{\pi}{5 m_{k}}\right) .\end{cases}
\end{aligned}
$$

Furthermore,

$$
\begin{gathered}
h(x)=\sum_{k=1}^{\infty} g_{k}(x) ; \\
p(y)= \begin{cases}\frac{1}{\ln (2 \pi / y) \ln (2)(2 \pi / y)}, & y \in\left(0 ; \frac{\pi}{2}\right], \\
\frac{1}{\ln (2 \pi /(\pi-y)) \ln ^{(2)}(2 \pi /(\pi-y))}, & y \in\left[\frac{\pi}{2} ; \pi\right) ;\end{cases} \\
f(x, y)= \begin{cases}h(x) p(y), & (x, y) \in(0 ; \pi)^{2}, \\
0, & (x, y) \in[-\pi ; \pi]^{2} \backslash(0 ; \pi)^{2} .\end{cases}
\end{gathered}
$$

Finally, outside the square $[-\pi ; \pi]^{2}$, we extend the function $f$ by periodicity with the period $2 \pi$ in each variable. It is easy to see that $f$ satisfies (26). 
From now on we set $m=2 m_{k}, n=n_{k}+1$. We have

$$
\begin{gathered}
\bar{\sigma}_{m n}^{\alpha, \beta}(0,0 ; f)-\bar{f}_{m n}(0,0)=1 / \pi^{2} \int_{0}^{\pi} \int_{0}^{\pi} f(x, y) \bar{K}_{m}^{\alpha}(x) \bar{K}_{n}^{\beta}(y) d y d x- \\
-1 /\left(4 \pi^{2}\right) \int_{1 / m}^{\pi} \int_{1 / n}^{\pi} f(x, y) \operatorname{ctg} \frac{x}{2} \operatorname{ctg} \frac{y}{2} d y d x= \\
=1 / \pi^{2} \int_{0}^{1 / m} \int_{0}^{1 / n} f(x, y) \bar{K}_{m}^{\alpha}(x) \bar{K}_{n}^{\beta}(y) d y d x+ \\
+1 / \pi^{2} \int_{0}^{1 / m} \int_{1 / n}^{\pi} f(x, y) \bar{K}_{m}^{\alpha}(x) \bar{K}_{n}^{\beta}(y) d y d x+ \\
+1 / \pi^{2} \int_{1 / m}^{\pi} \int_{0}^{1 / n} f(x, y) \bar{K}_{m}^{\alpha}(x) \bar{K}_{n}^{\beta}(y) d y d x+ \\
+1 / \pi^{2} \int_{1 / m}^{\pi} f(x, y)\left(\bar{K}_{m}^{\alpha}(x) \bar{K}_{n}^{\beta}(y)-1 / 4 \operatorname{ctg} \frac{x}{2} \operatorname{ctg} \frac{y}{2}\right) d y d x= \\
=\sum_{j=1}^{4} R_{j}(m, n) .
\end{gathered}
$$

Obviously,

$$
R_{1}(m, n)=o(1) \quad(m, n \rightarrow \infty)
$$

Then,

$$
\begin{aligned}
R_{2}(m, n) & =1 / \pi^{2} \int_{0}^{1 / m} \int_{1 / n}^{\pi} f(x, y) \bar{K}_{m}^{\alpha}(x) \frac{1}{2} \operatorname{ctg} \frac{y}{2} d y d x- \\
& -1 / \pi^{2} \int_{0}^{1 / m} \int_{1 / n}^{\pi} f(x, y) \bar{K}_{m}^{\alpha}(x) H_{n}^{\beta}(y) d y d x= \\
& =R_{2}^{\prime}(m, n)+R_{2}^{\prime \prime}(m, n),
\end{aligned}
$$

where

$$
H_{n}^{\alpha}(t)=\frac{1}{A_{n}^{\alpha}} \sum_{\nu=0}^{n} A_{\nu}^{\alpha-1} \frac{\cos (\nu+1 / 2) t}{2 \sin (t / 2)}
$$

The following estimates hold true (see [2], (5.12)):

$$
\begin{gathered}
\left|\bar{K}_{n}^{\alpha}(t)\right| \leq n, \quad|t| \leq \pi \\
\left|H_{n}^{\alpha}(t)\right| \leq C(\alpha) n^{-\alpha} t^{-(\alpha+1)}, \quad 1 / n \leq|t| \leq \pi
\end{gathered}
$$


Now we have

$$
\begin{gathered}
\int_{1 / n}^{\pi} p(y) \frac{1}{2} \operatorname{ctg} \frac{y}{2} d y=\int_{1 / n}^{\pi / 2} p(y) \frac{1}{2} \operatorname{ctg} \frac{y}{2} d y+ \\
+\int_{\pi / 2}^{\pi} p(y) \frac{1}{2} \operatorname{ctg} \frac{y}{2} d y=U_{1}(n)+U_{2}(n) .
\end{gathered}
$$

Now,

$$
\begin{gathered}
U_{1}(n)=-C_{1} \int_{1 / n}^{\pi / 2} \frac{d(2 \pi y)}{2 \pi y \ln (2 \pi y) \ln |\ln (2 \pi y)|} \leq \\
\leq C_{2} \ln ^{(3)} n \leq C_{3} m_{k} \ln m_{k} ; \\
\left|U_{2}(n)\right| \leq M .
\end{gathered}
$$

(31) and (35)-(37) yield

$$
\left|R_{2}^{\prime}(m, n)\right| \leq \frac{C}{\ln m_{k+1} \ln ^{(2)} m_{k+1}} m_{k} \ln m_{k} .
$$

As to $R_{2}^{\prime \prime}(m, n)$, we have

$$
\begin{gathered}
\left|R_{2}^{\prime \prime}(m, n)\right| \leq \frac{C(\beta)}{\ln m_{k+1} \ln ^{(2)} m_{k+1}} \int_{0}^{1 / m} \int_{1 / n}^{\pi} \frac{m}{n^{\beta} y^{\beta+1}} d y d x \leq \\
\leq \frac{C(\beta)}{\ln m_{k+1} \ln ^{(2)} m_{k+1}} .
\end{gathered}
$$

(31), (38) and (39) yield

$$
\left|R_{2}(m, n)\right| \leq \frac{C(\beta)}{\ln m_{m+1} \ln ^{(2)} m_{k+1}} m_{k} \ln m_{k} .
$$

Analogously

$$
R_{3}(m, n)=R_{3}^{\prime}(m, n)+R_{3}^{\prime \prime}(m, n) .
$$

Now,

$$
\begin{gathered}
\left|R_{3}^{\prime}(m, n)\right| \leq \frac{C}{\ln n \ln ^{(2)} n} \ln m \\
\left|R_{3}^{\prime \prime}(m, n)\right| \leq \frac{C(\alpha)}{\ln n \ln ^{(2)} n} .
\end{gathered}
$$

(41)-(43) yield

$$
\left|R_{3}(m, n)\right|=o(1) \quad(m, n \rightarrow \infty) .
$$


Now let us consider $R_{4}(m, n)$. We break it into 4 parts as follows

$$
\begin{aligned}
R_{4}(m, n)= & \left(\int_{1 / m}^{1 / m^{\tau}} \int_{1 / n}^{1 / n^{\tau}}+\int_{1 / m}^{1 / m^{\tau}} \int_{1 / n^{\tau}}^{\pi}+\int_{1 / m^{\tau}}^{\pi} \int_{1 / n}^{1 / n^{\tau}}+\right. \\
+ & \left.\int_{1 / m^{\tau}}^{\pi} \int_{1 / n^{\tau}}^{\pi}\right) 1 / \pi^{2} f(x, y)\left(\bar{K}_{m}^{\alpha}(x) \bar{K}_{n}^{\beta}(y)-\right. \\
& \left.-\frac{1}{4} \operatorname{ctg} \frac{x}{2} \operatorname{ctg} \frac{y}{2}\right) d y d x=\sum_{j=1}^{4} I_{j}(m, n),
\end{aligned}
$$

where $1 / 2 \leq \tau<1$.

We have

$$
\begin{gathered}
\left|I_{4}(m, n)\right| \leq \\
\leq C(\alpha, \beta) \int_{1 / m^{\tau}}^{\pi} \int_{1 / n^{\tau}}^{\pi}\left(\frac{1}{m^{\alpha} x^{\alpha+1} y}+\frac{1}{n^{\beta} y^{\beta+1} x}+\frac{1}{m^{\alpha} n^{\beta} x^{\alpha+1} y^{\beta+1}}\right) d y d x .
\end{gathered}
$$

It is easy to see that

$$
\left|I_{4}(m, n)\right|=o(1) \quad(m, n \rightarrow \infty) .
$$

Now we estimate $I_{2}(m, n)$.

$$
\begin{gathered}
I_{2}(m, n)=\sum_{k=1}^{3} Q_{k}(m, n) . \\
\left|Q_{2}(m, n)\right|=\left|1 / \pi^{2} \int_{1 / m}^{1 / m^{\tau}} \int_{1 / n^{\tau}}^{\pi} f(x, y) \frac{-H_{n}^{\beta}(y)}{2 \operatorname{tg}(x / 2)} d y d x\right| \leq \\
\leq C(\beta) \max |f| \ln m \frac{n^{\beta \tau}}{n^{\beta}}
\end{gathered}
$$

and

$$
\begin{gathered}
\left|Q_{2}(m, n)\right|=o(1) \quad(m, n \rightarrow \infty) \\
\left|Q_{3}(m, n)\right|=\left|1 / \pi^{2} \int_{1 / m}^{1 / m^{\tau}} \int_{1 / n^{\tau}}^{\pi} f(x, y) H_{m}^{\alpha}(x) H_{n}^{\beta}(y) d y d x\right|=o(1) \\
(m, n \rightarrow \infty)
\end{gathered}
$$

Next we will show that $\left|Q_{1}(m, n)\right| \rightarrow+\infty$ as $m, n \rightarrow \infty$.

We have $\frac{1}{m}<\frac{\pi}{6 m_{k}}<\frac{\pi}{5 m_{k}}<\frac{1}{m^{\tau}}$. Therefore

$$
Q_{1}(m, n)=1 / \pi^{2} \int_{\pi / 6 m_{k}}^{\pi / 5 m_{k}} h(x)\left(-H_{m}^{\alpha}(x)\right) d x \times
$$




$$
\times \int_{1 / n^{\tau}}^{\pi} p(y) \frac{1}{2 \operatorname{tg}(y / 2)} d y=Q_{1}^{(1)}(m) Q_{1}^{(2)}(n) .
$$

Since $m=2 m_{k}$, we have $\cos (i+1 / 2) x>0$ for $i=0,1, \ldots, m$ and $x \in\left[\frac{\pi}{6 m_{k}} ; \frac{\pi}{5 m_{k}}\right]$. Hence $\left(-H_{m}^{\alpha}(x)\right)<0$ (see (32)). Therefore we have

$$
\begin{gathered}
\left|Q_{1}^{(1)}(m)\right|=1 / \pi^{2} \int_{\pi / 6 m_{k}}^{\pi / 5 m_{k}} H_{m}^{\alpha}(x) h(x) d x \geq \\
\geq \frac{C}{A_{m}^{\alpha}} \int_{21 \pi / 120 m_{k}}^{23 \pi / 120 m_{k}} \sum_{i=0}^{m} A_{i}^{\alpha-1} \frac{\cos \frac{9 \pi}{20}}{x} h(x) d x \geq \\
\geq \frac{C}{A_{m}^{\alpha}} A_{m}^{\alpha} \frac{1}{\ln m_{k} \ln ^{(2)} m_{k}} \int_{21 \pi / 120 m_{k}}^{23 \pi / 120 m_{k}} \frac{d x}{x} \geq \frac{C}{\ln m_{k} \ln ^{(2)} m_{k}} .
\end{gathered}
$$

As to $Q_{1}^{(2)}(n)$, we have (analogously to $(36)$ )

$$
Q_{1}^{(2)}(n) \geq C m_{k} \ln m_{k}
$$

(50)-(52) imply

$$
\left|Q_{1}(m, n)\right| \geq \frac{C}{\ln m_{k} \ln ^{(2)} m_{k}} m_{k} \ln m_{k} .
$$

(28), (40) and (53) yield

$$
\left|Q_{1}(m, n)\right|-R_{2}(m, n) \rightarrow+\infty
$$

as $m, n \rightarrow \infty$ (for $\left.m=2 m_{k}, n=n_{k}+1\right)$.

From (47)-(49) and (54) we obtain

$$
\left|I_{2}(m, n)\right|-R_{2}(m, n) \rightarrow+\infty \quad(m, n \rightarrow \infty) .
$$

Now let us consider $I_{3}(m, n)$. As in the case of $I_{2}(m, n)$, we have

$$
I_{3}(m, n)=\sum_{\nu=1}^{3} J_{\nu}(m, n) .
$$

Then

$$
\begin{gathered}
\left|J_{1}(m, n)\right|=1 / \pi^{2}\left|\int_{1 / m^{\tau}}^{\pi} h(x)\left(-H_{m}^{\alpha}(x)\right) d x \int_{1 / n}^{1 / n^{\tau}} \frac{p(y)}{2 \operatorname{tg}(y / 2)} d y\right| \leq \\
\leq C(\alpha) \frac{m^{\alpha \tau}}{m^{\alpha}}\left(\ln ^{(3)} n^{\tau}-\ln ^{(3)} n\right)=\frac{C(\alpha)}{m^{\alpha(1-\tau)}} \ln \frac{\ln ^{(2)} n^{\tau}}{\ln ^{(2)} n}
\end{gathered}
$$




$$
\begin{gathered}
\left|J_{2}(m, n)\right|=\left|1 / \pi^{2} \int_{1 / m^{\tau}}^{\pi} \frac{h(x)}{2 \operatorname{tg}(x / 2)} d x \int_{1 / n}^{1 / n^{\tau}} p(y)\left(-H_{n}^{\beta}(y)\right) d y\right| \leq \\
\leq \frac{C(\beta) \ln m}{\ln n \ln ^{(2)} n} \int_{1 / n}^{1 / n^{\tau}} \frac{d y}{n^{\beta} y^{\beta+1}} ; \\
\left|J_{3}(m, n)\right|=\left|1 / \pi^{2} \int_{1 / m^{\tau}}^{\pi} \int_{1 / n}^{1 / n^{\tau}} h(x) p(y) H_{m}^{\alpha}(x) H_{n}^{\beta}(y) d y d x\right| \leq \\
\leq C(\alpha, \beta) \frac{m^{\alpha \tau}}{m^{\alpha}} \frac{n^{\beta}}{n^{\beta}} .
\end{gathered}
$$

(56)-(59) yield

$$
I_{3}(m, n)=o(1) \quad(m, n \rightarrow \infty) .
$$

Now we consider $I_{1}(m, n)$. As above,

$$
I_{1}(m, n)=\sum_{j=1}^{3} T_{j}(m, n) .
$$

We have

$$
\begin{aligned}
\left|T_{1}(m, n)\right| & =1 / \pi^{2}\left|\int_{1 / m}^{1 / m^{\tau}} \int_{1 / n}^{1 / n^{\tau}} h(x) p(y)\left(-H_{m}^{\alpha}(x)\right) \frac{1}{2} \operatorname{ctg} \frac{y}{2} d y d x\right| \leq \\
& \leq \frac{C_{1}(\alpha)}{\ln m_{k} \ln ^{(2)} m_{k}} \frac{C_{2}(\alpha)}{\ln n_{k} \ln ^{(2)} n_{k}} \frac{m^{\alpha}}{m^{\alpha}} \ln n ; \\
\left|T_{2}(m, n)\right| & =1 / \pi^{2}\left|\int_{1 / m}^{1 / m^{\tau}} \int_{1 / n}^{1 / n^{\tau}} h(x) p(y)\left(-H_{n}^{\beta}(y)\right) \frac{1}{2} \operatorname{ctg} \frac{x}{2} d y d x\right| \leq \\
& \leq \frac{C_{1}(\beta)}{\ln m_{k} \ln ^{(2)} m_{k}} \frac{C_{2}(\beta)}{\ln n_{k} \ln ^{(2)} n_{k}} \frac{n^{\beta}}{n^{\beta}} \ln m ; \\
\left|T_{3}(m, n)\right| & =1 / \pi^{2}\left|\int_{1 / m}^{1 / m^{\tau}} \int_{1 / n}^{1 / n^{\tau}} h(x) p(y) H_{m}^{\alpha}(x) H_{n}^{\beta}(y) d y d x\right| \leq \\
& \leq \frac{C_{1}(\alpha, \beta)}{\ln m_{k} \ln ^{(2)} m_{k}} \frac{C_{2}(\alpha, \beta)}{\ln n_{k} \ln ^{(2)} n_{k}} \frac{m^{\alpha} n^{\beta}}{m^{\alpha} n^{\beta}} .
\end{aligned}
$$

(61)-(64) yield

$$
I_{1}(m, n)=o(1) \quad(m, n \rightarrow \infty)
$$

(we remind once more that $m=2 m_{k}, n=n_{k}+1$ ).

Finally, (29), (30), (44)-(46), (55), (60) and (65) prove the Theorem 2 in the case $n=2$ and $\alpha, \beta \in(0 ; 1)$. 
For $\alpha=1$ we have

$$
H_{n}^{1}(t)=\frac{\sin (n+1) t}{(n+1)\left(2 \sin \frac{t}{2}\right)^{2}}
$$

and an estimate analogous to (34) holds true.

Now we consider the case when $\alpha>1$. Using the method represented in [4], p.507, we obtain

$$
\begin{aligned}
H_{n}^{\alpha}(t) & =\frac{1}{A_{n}^{\alpha} 2 \sin \frac{t}{2}} \operatorname{Re}\left\{\frac{e^{i(n+1 / 2) t}}{\left(1-e^{-i t}\right)^{\alpha}}-e^{-\frac{1}{2} i t} \sum_{m=1}^{d} A_{n}^{\alpha-m}\left(1-e^{-i t}\right)^{-m}-\right. \\
& \left.-\sum_{m=n+1}^{\infty} A_{m}^{\alpha-d-1} e^{-i(m-n-1 / 2) t}\left(1-e^{-i t}\right)^{-d}\right\}
\end{aligned}
$$

where $d=[\alpha]$.

Taking the real part we obtain that the first term of the finite sum is 0 . Therefore if $[\alpha]=1$ we apply once more the Abel transformation to the infinite sum in (67) and obtain

$$
\begin{aligned}
H_{n}^{\alpha}(t) & =\frac{\cos \left(\left(n+\frac{1}{2}+\frac{\alpha}{2}\right) t-\frac{\pi \alpha}{2}\right)}{A_{n}^{\alpha}\left(2 \sin \frac{t}{2}\right)^{1+\alpha}}-\frac{(1-\alpha) \alpha \cos (t / 2)}{8(n+1)(n+\alpha)\left(\sin \frac{t}{2}\right)^{3}}- \\
& -\frac{1}{A_{n}^{\alpha}} \sum_{m=1}^{\infty} A_{m+n+1}^{\alpha-3} \frac{\sin (m-1) t}{\left(2 \sin \frac{t}{2}\right)^{3}}
\end{aligned}
$$

Then, again, we have an estimate analogous to (34), which enables us to fulfil the proof. Namely,

$$
\left|H_{n}^{\alpha}(t)\right| \leq \frac{C_{1}(\alpha)}{n^{\alpha} t^{\alpha+1}}+\frac{C_{2}(\alpha)}{n^{2} t^{3}} .
$$

If $[\alpha]=2$ without further transformation we obtain

$$
\begin{aligned}
H_{n}^{\alpha}(t) & =\frac{\cos \left(\left(n+\frac{1}{2}+\frac{\alpha}{2}\right) t-\frac{\pi \alpha}{2}\right)}{A_{n}^{\alpha}\left(2 \sin \frac{t}{2}\right)^{1+\alpha}}+\frac{(\alpha-1) \alpha \cos (t / 2)}{8(n+\alpha-1)(n+\alpha)\left(\sin \frac{t}{2}\right)^{3}}+ \\
& +\frac{1}{A_{n}^{\alpha}} \sum_{m=1}^{\infty} A_{m+n}^{\alpha-3} \frac{\cos (m-3 / 2) t}{\left(2 \sin \frac{t}{2}\right)^{3}}
\end{aligned}
$$

and, again, (69) holds true.

Analogous equations may be obtained if $[\alpha] \geq 3$.

Now, when $\alpha=1$ and $\beta=1$, we use (66). If $\alpha=1$ and $\beta<1$ (or vice versa), we use (34) and (66). If $\alpha=1$ and $\beta>1$ (or vice versa), we use (66) and (67) (for the corresponding $d$ ). If $\alpha>1$ and $\beta<1$ (or vice versa), we use again (67) (for the corresponding $d$ ) and (34). 
In the $n$-dimensional case we define $\mathrm{f}$ as follows. We set

$$
g_{k}(x)= \begin{cases}\frac{m_{k}=2^{2^{2^{k}}}, \quad(k \in \mathbb{N}) ;}{\frac{1}{\ln ^{n-1}\left(1 /\left(x-\pi /\left(6 m_{k}\right)\right)\right) \ln (n)}\left(1 /\left(x-\pi /\left(6 m_{k}\right)\right)\right)}, & \frac{\pi}{6 m_{k}}<x \leq \frac{11 \pi}{60 m_{k}}, \\ \frac{1}{\ln ^{n-1}\left(1 /\left(\pi /\left(5 m_{k}\right)-x\right)\right) \ln ^{(n)}\left(1 /\left(\pi /\left(5 m_{k}\right)-x\right)\right)}, & \frac{11 \pi}{60 m_{k}} \leq x<\frac{\pi}{5 m_{k}}, \\ 0, & x \in(0 ; \pi) \backslash\left(\frac{\pi}{6 m_{k}} ; \frac{\pi}{5 m_{k}}\right),\end{cases}
$$

Again

$$
h(x)=\sum_{k=1}^{\infty} g_{k}(x) .
$$

Then,

$$
=\frac{p\left(x_{2}, \ldots, x_{n}\right)=}{\ln ^{n-1} \frac{1}{\prod_{i=2}^{n}\left(\pi / 2-\left|\pi / 2-x_{i}\right|\right)} \ln ^{(n)} \frac{(2 \pi)^{n-1}}{\prod_{i=2}^{n}\left(\pi / 2-\left|\pi / 2-x_{i}\right|\right)}}
$$

for $\left(x_{2}, \ldots, x_{n}\right) \in(0 ; \pi)^{n-1}$.

And, finally

$$
f\left(x_{1}, \ldots, x_{n}\right)= \begin{cases}h\left(x_{1}\right) p\left(x_{2}, \ldots, x_{n}\right), & \left(x_{1}, \ldots, x_{n}\right) \in(0 ; \pi)^{n}, \\ 0, & \left(x_{1}, \ldots, x_{n}\right) \in[-\pi ; \pi]^{n} \backslash(0 ; \pi)^{n} .\end{cases}
$$

Outside $[-\pi ; \pi]^{n}$ we extend the function $f$ by periodicity with the period $2 \pi$ in each variable.

We observe, that functions of the $p\left(x_{2}, \ldots, x_{n}\right)$-type were for the first time introduced and applied in the works of L.Zhizhiashvili (see [1], [5]).

Remark 1. For the function $f\left(x_{1}, \ldots, x_{n}\right)$ a stronger condition than (26) holds true, namely,

$$
\omega_{i}(\delta ; f)_{C} \leq \frac{C(f, n)}{\ln ^{n-1}(1 / \delta) \ln ^{(n)}(1 / \delta)}, \quad i=\overline{1, n} .
$$

Remark 2. Results analogous to Theorem 1, the corollary and Theorem 2 hold true for the n-multiple Abel-Poisson summability method.

\section{REFERENCES}

1. L.V. Zhizhiashvili, Conjugate functions and trigonometric series. (Russian) Tbilisi University Press, Tbilisi, 1969. 
2. A. Zygmund, Trigonometric series, v.1. (Translated into the Russian) "Mir", Moscow, 1965; English original: Cambridge, 1959.

3. G.H. Hardy, J.E. Littlewood and G. Pólya, Inequalities. (Translated into the Russian) "IL", Moscow, 1949; English original: Cambridge, 1934.

4. M.M. Lekishvili, On approximation of periodic functions by $(C, \alpha)$ means. (Russian) Mat.Sb. 121(163)(1983), No.4(8), 499-509.

5. L.V. Zhizhiashvili, On some problems from the theory of simple and multiple trigonometric and orthogonal series. (Russian) Uspekhi Mat.Nauk 28(1973), No.2, 65-119.

(Received 25.02.1993, revized version 13.05.1993)

Author's address:

Faculty of Mechanics and Mathematics

I. Javakhishvili Tbilisi State University

2, University St., 380043 Tbilisi

Republic of Georgia 